\title{
Cellular coverage probability is independent of base station density under stochastic geometric models
}

\author{
Hamed Nassar, Gehad Taher, and El-Sayed El-Hady,
}

\begin{abstract}
Stochastic geometry (SG) has been extensively used to model cellular communications, under the assumption that the base stations (BS) are deployed as a Poisson point process in the Euclidean plane. This has spawned a huge number of articles over the past years for different scenarios, culminating in an equally huge number of expressions for the coverage probability in both the uplink (UL) and downink (DL) directions. The trouble is that those expressions include the BS density, $\lambda$, which we prove irrelevant in this article. We start by developing a SG model for a baseline cellular scenario, then prove that the coverage probability is independent of $\lambda$, contrary to popular belief.
\end{abstract}

Index Terms-Stochastic geometry; Cellular network; Base station density; Coverage probability; Independence

\section{INTRODUCTION}

$\mathbf{S}$ TOCHASTIC geometry (SG) has been used heavily in the late decade as a modelling tool in the field of wireless communications. It is particularly suited for modelling large scale wireless communication networks, where a network is treated as a realization (snapshot) of a spatial point process in the Euclidean plane [1]. In particular, it is a natural approach to describe node locations in randomly formed networks, e.g. ad hoc and cellular networks. It provides a natural way of examining macroscopic properties, by averaging over all potential locations of network nodes to obtain important performance characteristics, such as coverage probability. These locations in our context are the network elements at the time the snapshot is taken [2].

The most common function of SG in wireless communications is to characterize the signal to interference and noise ratio (SINR), which can then be used to calculate many cellular performance metrics, such as outage probability, coverage probability, spatial opportunity, spatial throughput, network throughput, medium access probability and spectral efficiency [3], in both downlink (DL) and uplink (UL) directions. It should be noted, however, that in light the huge of influx of wireless emissions in recent years, the impact of noise now pales in comparison with interference [4]. As such, there is a growing trend (see, for example, [5], [6], [7], [8]) to replace SINR by SIR, and we will follow this trend in the present article.

The most commonly made assumption when SG is used in modelling cellular networks is that base stations (BS) are deployed in the Euclidean plane as a Poisson Point Process

H. Nassar is with the Department of Computer Science, Suez Canal University, Ismailia 41522, Egypt. e-mail: (nassar@ci.suez.edu.eg).

G. Taher and S. El-Hady is with Suez Canal University, Ismailia 41522, Egypt.

Manuscript received ; revised
(PPP), although many variant processes, e.g. Thomas cluster processes or Matérn cluster processes, have been suggested as well [9]. This model replaces the once popular hexagonal grid model, in which base stations were placed at the centers of the hexagonal lattices. It has been shown [10] that the PPP approach provides much more accurate results than the hexagonal grid model when both are used to model real world cellular installation. It has also been shown [11] that the PPP model gives lower bounds, whereas the hexagonal model gives upper bounds, of the coverage probability, which means that the former is safer to rely on.

SG cellular models that end up with coverage probability expressions involving the BS density, $\lambda$, abound. These models are developed for different scenarios, but have in common the consideration that the coverage probability is dependent on $\lambda$. Examples of such models for DL are [2], [4], [6], [8], [12], [13], [14], [15], [16], [17], [18], [19], [20], [21], [22], [23]. On the other hand, examples of such models for DL are [2], [4], [7], [24], [25], [26], [20], [27], [18], [19], [21], [22], [23]. We prove in this article two theorems that refute this consideration. Specifically, we prove that under the SG model, the coverage probability is independent of $\lambda$, both in DL and UL.

To set the stage for these proofs, however, we will first develop a SG model for a baseline cellular scenario, then use the model to derive two expressions, including $\lambda$, for the coverage probability in DL and UL. Finally, we give two theorems that show show that $\lambda$ is superfluous.

It is worth mentioning that the notion that the coverage probability is independent of $\lambda$ has only been alluded to sparingly previously. The authors of [12] in the context of analyzing DL coverage probability of millimeter-wave cellular networks noted that "coverage does not scale with BS density." Also, the authors of [24], while analyzing UL FPC, noted that coverage is " invariant to the density of deployment of BSs when the shadowing is mild and power control is fractional." To the best of our knowledge, the present article is the first to provide a rigorous proof that coverage probability is independent of BS density.

The rest of the article is organized as follows. In Section II we develop SG models for DL and UL, and use these models to prove that the coverage probability is independent of the BS density $\lambda$. Section III has the conclusions.

\section{SySTEM MOdEL}

The key aspect of the present study is that all the BSs are located according to a PPP $\Phi$, which effectively means they are randomly scattered in the Euclidean plane with independent 
locations [9]. In the present article, we consider orthogonal within each cell, meaning that in each cell there can be only one active UE on any time/frequency resource. Accordingly, Figure 1 is a snapshot of the UEs that are active on the same frequency in all the cells at the same time. As for the entire network, every BS-UE pair is operating on the same resource, hence is the interference that we are going to characterize.

Some definitions used throughout the article are now in order.

Definition 1 (BS-UE association): BS-UE association is the assignment of a UE to a $\mathrm{BS}$, for both to establish a communications session.

Definition 2 (Serving BS): Once a UE is associated with a BS, the latter is said to be the serving BS of the UE.

Definition 3 (Typical receiver): The typical receiver is the receiving device (UE or BS) where the SIR is to be assessed. It is always placed at the origin of the Euclidean plane in the model, or the origin of the simulation window in the simulation.

Definition 4 (Tagged transmitter): The tagged transmitter is transmitting device (UE or BS) associated with the typical receiver.

Definition 5 (Typical circle): The typical circle is the circle centered at the typical receiver and having the tagged transmitter on its circumference.

Definition 8 (Interferer): An interferer is a transmitter causing interference at the typical receiver. That is, it is any transmitter in the network other than the tagged transmitter.

Definition 9 (Signal to Interference Ratio (SIR)): The quotient of the signal at the typical receiver and the sum of all interferences at the typical receiver.

As per Definitions 3 and 4, in DL the typical receiver is a UE and the tagged transmitter is a BS, whereas in UL the typical receiver is a $\mathrm{BS}$ and the tagged transmitter is a UE.

A UE will associate with the BS nearest it. We will denote the distance between the two elements of a BS-UE pair throughout by $R$. It can then be shown that $R$ is a random variable (RV) with the Rayleigh distribution

$$
f_{R}(r)=2 \lambda \pi r e^{-\lambda \pi r^{2}}, \quad r \geq 0
$$
I]

The notation used throughout the article is provided in Table

TABLE I: Notation used in the model.

\begin{tabular}{cl}
\hline \hline Parameter & Description \\
\hline BS & Base station \\
UE & User Equipment (can be a mobile phone, tablet, laptop, etc.) \\
$\Phi$ & Poisson point process (PPP) of BSs \\
$\Psi$ & Point process of UEs (not Poisson) \\
$\lambda$ & Density of BS (per $\left.\mathrm{m}^{2}\right)$, i.e. intensity of PPP $\Phi$ \\
$\alpha$ & Path-loss exponent (per m) \\
SIR & Signal to interference ratio (dB) \\
$\xi$ & SIR threshold (dB) \\
$G$ & Rayleigh channel gain of tagged transmitter $(G \sim \operatorname{Exp}(1))$ \\
$\mathrm{p}$ & Transmit power (Watts) \\
$p_{d}$ & DL coverage probability \\
$p_{u}$ & UL coverage probability \\
\hline
\end{tabular}

Referring to Figure 11 the typical receiver resides at the origin, and the tagged transmitter resides on the perimeter of the typical circle. In part 1a, we see the DL model, where the typical receiver is a $\mathrm{UE}$, and the tagged transmitter is a $\mathrm{BS}$ at distance $R$. All the BSs outside the typical circle are interferers to the typical UE. In part $1 \mathrm{~b}$, we can see the UL model, where the typical receiver is a $\mathrm{BS}$, and the tagged transmitter is a UE at distance $R$. All the UEs except the tagged are interferers to the typical BS.

Random channel effects are incorporated by multiplicative RVs, namely $G$ for the signal and $G_{i}$ for each interferer $i$. For simplicity we assume small-scale Rayleigh fading, and assume $G$ and the $G_{i}$ iid RVs following an exponential distribution with mean 1. In addition, we assume that signals attenuate with distance according to the standard power-law path loss propagation model, with path loss exponent $\alpha>2$. That is, the average received power at distance $r$ from a transmitter of power $p$ is $p r^{-\alpha}$.

\section{A. Downlink model}

The key assumptions of the DL system model are:

- BSs are located according to a homogeneous PPP $\Phi$ of intensity $\lambda$ in the Euclidean plane.

- A UE wishing to start a communications session associates with the BS that is closer to it than any other BS in the cellular network (association rule.)

- The BS transmits at a fixed power $p$ to a designated UE on a particular time-frequency resource, i.e. orthogonal communications within the cell. The consequence of orthogonality is that the UE sees interference from all the BSs in the plane, except its serving BS.

- Random channel effects are incorporated by a multiplicative RVs $G$ for the signal and $G_{z}$ for every interferer at $z$. For simplicity we assume these all to correspond to Rayleigh fading with mean 1 , so $G$ and the $G_{z}$ are iid RVs, having exponential distribution with mean 1 .

Let $I_{d}$ denote the interference experienced at the typical UE. The interference is due to every BS $z$ in the plane, except the tagged BS, denoted by $\mathfrak{b}$, at distance $D_{z}$ from the typical UE, as shown in Figure $1 \mathrm{a}$. That is, the interference $I_{d}$ is created by a PPP with intensity $\lambda$ outside the typical circle, and is given by

$$
I_{d}=\sum_{z \in \Phi \backslash\{\mathfrak{b}\}} p G_{z} D_{z}^{-\alpha},
$$

where $p$ is the power of the BS at point $z$, and $D_{z}$ is a RV representing the distance from the BS at $z \mathrm{a}$ and the typical UE. The SIR at the typical UE is then given by

$$
\operatorname{SIR}_{\mathrm{UE}}=\frac{p G R^{-\alpha}}{I_{d}}
$$

The goal now is to derive the DL coverage probability $p_{d}$, which is exactly the complementary cumulative distribution function (CCDF) of SIR over the entire network (Recall that the CDF gives $\mathbb{P}[\operatorname{SIR} \leq \xi]$ ). The coverage probability can also be visualized as the probability that a randomly chosen user can achieve a target SIR $\xi$, the average fraction of users who 
(a)

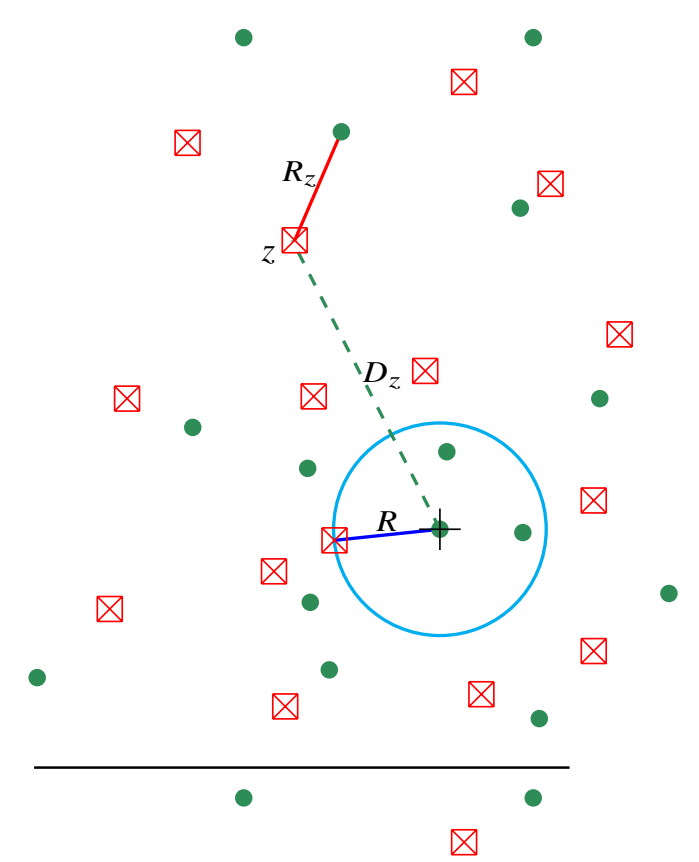

(b)

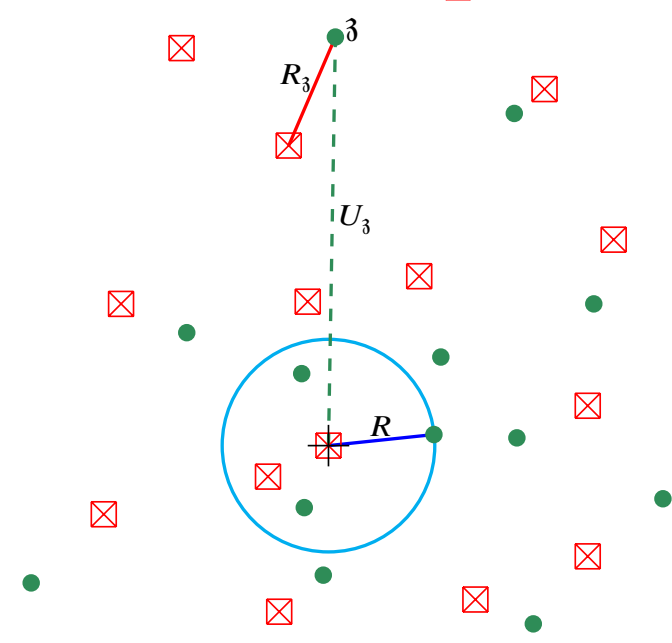

Fig. 1: Stochastic geometric models for assessing the SIR at a typical receiver at the origin of a cellular network. (a) The DL model, where the typical receiver is a UE. The typical circle defines an exclusion zone, as it cannot contain a BS inside. The BSs outside the typical circle, which cause interference at the typical UE, form a PPP $\Phi$. (b) The UL model, where the typical receiver is a BS. The typical circle does not define an exclusion zone, as it can contain UEs inside. All the UEs form a PP $\Psi$. Since the UEs are satellites to their serving BSs, as per the association rule, $\Psi$ is not Poisson, a major challenge to the analysis. We mitigate this challenge by relocating each UE, except the tagged, to the position of its serving BS. That is, we relocate all the interferers.

at any time achieve SIR $\xi$, or the average fraction of network area that is in "coverage" at any time.

We start by invoking the concept of total probability, using (2) and (3), to get

$$
p_{d \mid R}=\mathbb{P}\left[\operatorname{SIR}_{\mathrm{UE}}>\xi\right]
$$

$$
\begin{array}{lll} 
& = & \mathbb{P}\left[\frac{p G R^{-\alpha}}{I_{d}}>\xi\right] \\
= & \mathbb{P}\left[G>\frac{\xi}{p} R^{\alpha} I_{d}\right] \\
\stackrel{(\mathrm{a})}{=} & \mathbb{E}_{I_{d}}\left[\mathbb{P}\left[G>\frac{\xi}{p} R^{\alpha} I_{d}\right]\right] \\
\stackrel{(\mathrm{b})}{=} & \mathbb{E}_{I_{d}}\left[e^{-\frac{\xi}{p} R^{\alpha} I_{d}}\right] \\
\stackrel{(\mathrm{c})}{=} & & \mathcal{L}_{I_{d}}\left(\frac{\xi}{p} R^{\alpha}\right)
\end{array}
$$

where

$$
\mathcal{L}_{A}(s)=\int_{0}^{\infty} e^{-s t} f_{A}(t) d t=\mathbb{E}\left[e^{-s A}\right]
$$

is the Laplace transform of the RV $I_{d}$ conditioned on the RV $R$ between the typical UE and the tagged BS. In (a) we utilized the fact that we can write a probability $\mathbb{P}[A>B)]$ as $\mathbb{E}_{B}[\mathbb{P}[A>B]]$ (or $\mathbb{E}_{A}[\mathbb{P}[A>B]]$ ), in (b) we benefited from the fact that $G \sim \exp (1)$, i.e. $f_{G}(r)=e^{-r}$, and in (c) we used the Laplace transform definition (5).

Clearly, the DL coverage probability $p_{d}$ in (4) is conditioned on $R$, the distance between the typical UE and the tagged BS. We will now embark on deconditioning $p_{d}$. Since $R$ is the distance between the typical UE and the closest BS (the tagged $\mathrm{BS})$, it is Rayleigh distributed, i.e. and $f_{R}(r)=2 \lambda \pi r e^{-\lambda \pi r^{2}}$ from (1). Further, $R$ ranges from an arbitrarily small positive real number greater than 0 (to exclude the typical UE) to $\infty$. Thus, the conditional coverage probability

$$
\begin{aligned}
p_{d \mid R} & =\mathbb{E}_{R}\left[p_{d \mid R}\right] \\
& =\mathbb{E}_{R}\left[\mathcal{L}_{I_{d}}\left(\frac{\xi}{p} R^{\alpha}\right)\right] \\
& =\int_{0}^{\infty} \mathcal{L}_{I_{d}}\left(\frac{\xi}{p} r^{\alpha}\right) f_{R}(r) d r \\
& =2 \lambda \pi \int_{0}^{\infty} e^{-\lambda \pi r^{2}} \mathcal{L}_{I_{d}}\left(\frac{\xi}{p} r^{\alpha}\right) r d r
\end{aligned}
$$

Next, we will embark on finding the Laplace transform $\mathcal{L}_{I_{d}}$ of the DL interference $I_{d}$. Using (2) and (5), we get

$$
\begin{aligned}
\mathcal{L}_{I_{d}}(s)= & \mathbb{E}\left[e^{-s I_{d}}\right] \\
= & \mathbb{E}_{\Phi, G_{z}}\left[e^{-s \sum_{z \in \Phi \backslash\{\mathfrak{b}\}} p G_{z} D_{z}^{-\alpha}}\right] \\
= & \mathbb{E}_{\Phi, G_{z}}\left[\prod_{z \in \Phi \backslash\{\mathfrak{b}\}} e^{-s p G_{z} D_{z}^{-\alpha}}\right] \\
\stackrel{(\mathrm{a})}{=} & \mathbb{E}_{\Phi}\left[\prod_{z \in \Phi \backslash\{\mathfrak{b}\}} \mathbb{E}_{G_{z}}\left[e^{-s p G_{z} D_{z}^{-\alpha}}\right]\right] \\
\stackrel{(\mathrm{b})}{=} & \mathbb{E}_{\Phi}\left[\prod_{z \in \Phi \backslash\{\mathfrak{b}\}} \mathcal{L}_{G_{z}}\left(s p D_{z}^{-\alpha}\right)\right] \\
& \stackrel{\operatorname{(c)}}{=} \quad \\
& \exp \left(-\lambda \int_{\mathbb{R}^{2} \backslash D(o, r)}\left(1-\mathcal{L}_{G_{z}}\left(s p D_{z}^{-\alpha}\right)\right)\right)
\end{aligned}
$$

where $D(o, r)$ is a disc centered at the origin and has a radius $r$. In (a) we benefited from the independence of the $G_{z}$, which are iid and in (b) we used the definition (5) of the Laplace transform. In (c), to decondition on $D_{z}$ which is 
distributed differently for each point $z$ of the PPP, we invoked the PGFL $\mathbb{E}_{\Phi}\left[\prod_{z \in \Phi} f(x)\right]$, with $f(x)=\mathcal{L}_{G_{z}}\left(s p x^{-\alpha}\right)$, of the PPP $\Phi$. Switching to polar coordinates, with the interferer now at $(x, \theta) \in \mathbb{R}^{2}$, then using the fact that $G_{z} \sim \exp (1)$, i.e. $f_{G_{z}}(t)=e^{-t}$, then Using (7) yields

$$
\begin{aligned}
\mathcal{L}_{I_{d}}(s) & =\exp \left(-\lambda \int_{0}^{2 \pi} \int_{r}^{\infty}\left(1-\mathcal{L}_{G_{z}}\left(s p x^{-\alpha}\right)\right) x d x d \theta\right) \\
& =\exp \left(-2 \pi \lambda \int_{r}^{\infty}\left(\frac{s p x^{-\alpha}}{1+s p x^{-\alpha}}\right) x d x\right)
\end{aligned}
$$

For use in (6), we write this result as

$$
\begin{aligned}
\mathcal{L}_{I_{d}}\left(\frac{\xi}{p} r^{\alpha}\right) & =\exp \left(-2 \pi \lambda \int_{r}^{\infty}\left(\frac{\left(\frac{\xi}{p} r^{\alpha}\right) p x^{-\alpha}}{1+\left(\frac{\xi}{p} r^{\alpha}\right) p x^{-\alpha}}\right) x d x\right) \\
& =\exp \left(-\pi \lambda r^{2} \xi^{\frac{2}{\alpha}} \int_{\xi^{-2 / \alpha}}^{\infty} \frac{1}{1+u^{\alpha / 2}} d u\right)
\end{aligned}
$$

where $u=(x / r)^{2} \xi^{-\frac{2}{\alpha}}$. Substituting this in $(6)$, we get

$$
p_{d}=2 \tilde{\lambda} \int_{0}^{\infty} e^{-\widetilde{\lambda} r^{2}} e^{-\tilde{\lambda} r^{2} \sqrt[k]{\xi} \int_{\sqrt[1]{\xi}}^{\infty} \frac{1}{1+u^{k}} d u} r d r
$$

where $\tilde{\lambda}=\lambda \pi$ and $\kappa=\alpha / 2$.

From (9), it appears that the DL coverage probability $p_{d}$ is dependent on the BS density $\lambda$. However, this dependence is false, as we will show in the next Theorem.

Theorem 1: Under the stochastic geometric model of the cellular DL system, the DL coverage probability $p_{d}$ is independent of the BS density $\lambda$.

Proof: The proof is attained through two changes of variables. Starting with 99, use the substitution $x=r^{2}$ to get

$$
\begin{aligned}
p_{d} & =2 \widetilde{\lambda} \int_{0}^{\infty} e^{-\tilde{\lambda} r^{2}} e^{-\tilde{\lambda} r^{2} \xi^{\frac{2}{\alpha}} \int_{\xi^{-\frac{2}{\alpha}}}^{\infty} \frac{1}{1+u^{\kappa}} d u} r d r \\
& =\tilde{\lambda} \int_{0}^{\infty} e^{-\tilde{\lambda} x\left(1+\sqrt[k]{\xi} \int_{\frac{1}{\sqrt{\xi}}}^{\infty} \frac{1}{1+u^{\kappa}} d u\right)} d x
\end{aligned}
$$

Now use the substitution $z=\widetilde{\lambda} x$ to get

$$
\begin{aligned}
p_{d} & =\int_{0}^{\infty} e^{-z\left(1+\sqrt[k]{\xi} \int_{\frac{1}{\sqrt[k]{\xi}}}^{\infty} \frac{1}{1+u^{\kappa}} d u\right)} d z \\
& =\frac{1}{1+\sqrt[k]{\xi} \int_{\frac{1}{\sqrt[k]{\xi}}}^{\infty} \frac{1}{1+u^{\kappa}} d u}
\end{aligned}
$$

where $\lambda$ has totally disappeared, proving the theorem.

\section{B. Uplink model}

Referring to Figure $1 \mathrm{~b}$, the net interference at the typical BS is the sum of the received transmissions from all the UEs (including those inside the typical circle) except the tagged. For each UE $\mathfrak{z} \in \Psi$, we denote its distance to its serving BS by $R_{\mathfrak{z}}$.

Here, the set of interferers are the points of $\Psi$, which is not a PPP. To get around this difficutly, note that each point of $\Psi$ is associated to a point in the PPP $\Phi$ of BSs, which we used above in the down link analysis. Thus, we can approximate the "spatial" average of the former to be the latter. Consequently, we can consider the locations $\Psi$ of the interfering UEs by the locations $\Phi$ of the BSs. Specifically, for calculating the interference, we will consider that each interfering UE is placed exactly at its serving BS's location. Referring to Figure $1 \mathrm{~b}$, we will employ the distance $R_{\mathfrak{z}}$ between this UE and its serving BS to calculate its emitted power. We will then consider this as interference at the typical BS at distance $D_{z}$ away, not $U_{3}$.

We will assume fractional power control (FPC), where each user equipment (UE) adjusts its power level in the UL direction under the control of its serving BS [7]. FPC leads to amplifying the transmit power $p$ at the UE based on its distance to the serving BS. If the distance is $R$ and the FPC factor is $\epsilon$, with values in $[0,1]$, then $p$ is amplified by $R^{\epsilon \alpha}$ to offset the path loss, which is $R^{-\alpha}$, where $\alpha>2$ is the path loss exponent. Combining the effects of FPC, power loss and fading, the amount of power reaching the serving BS from a $\mathrm{UE}$ is $p G R^{-\alpha(1-\epsilon)}$.

Referring to Figure $1 \mathrm{~b}$, the RV $R_{\mathfrak{3}}$ is upper bounded by $U_{3}$, otherwise the sample UE at $z$ would associate with the typical BS. Accordingly,

$$
I_{u}=\sum_{\mathfrak{z} \in \Psi \backslash\{\mathfrak{u}\}} p G_{\mathfrak{z}} R_{\mathfrak{z}}^{\alpha \epsilon} U_{\mathfrak{z}}^{-\alpha}
$$

In the UL, the SIR of the typical BS, at distance $R$ from the tagged UE, is

$$
\mathrm{SIR}_{\mathrm{BS}}=\frac{p G R^{-\alpha(1-\epsilon)}}{I_{u}}
$$

Consequently, the probability $p_{u}$ of UL coverage is

$$
p_{u}=\mathbb{P}\left[\operatorname{SIR}_{\mathrm{BS}}>\xi\right] .
$$

Referring to Figure $1 \mathrm{~b}$, both $R$ and $R_{3}$ are Rayleigh distributed, i.e. $f_{R}(r)=\overline{f_{R_{\mathfrak{z}}}}(r)=2 \lambda \pi r e^{-\lambda \pi r^{2}}$. Thus, $R_{\mathfrak{z}}$ ranges from 0 to $R$, with $R$ ranging from an arbitrarily small positive real number to $\infty$. Now, the conditional UL coverage probability is defined as

$$
\begin{aligned}
p_{u \mid R} & =\mathbb{P}\left[\mathrm{SIR} \mathrm{BS}_{\mathrm{BS}} \xi\right] \\
& =\mathbb{E}\left[\mathbb{P}\left[\frac{p G R^{-\alpha(1-\epsilon)}}{I_{u}}>\xi\right]\right] \\
& =\mathbb{E}\left[\mathbb{P}\left[G>\xi p^{-1} R^{\alpha(1-\epsilon)} I_{u}\right]\right] \\
& \stackrel{(\mathrm{a})}{=} \mathbb{E}\left[e^{-\xi p^{-1} I_{u} R^{\alpha(1-\epsilon)}}\right] \\
& =\mathcal{L}_{I_{u}}\left(\xi p^{-1} R^{\alpha(1-\epsilon)}\right)
\end{aligned}
$$

where $\mathcal{L}_{I_{u}}$ is the Laplace transform of the distribution of the $I_{u}$ RV. In (a), we used the fact that $G \sim \exp (1)$, i.e. $f_{G}(x)=e^{-x}$, which implies that $\mathbb{P}[G>x]=e^{-x}$. Now, we decondition on $R$, getting

$$
\begin{aligned}
p_{u} & =\left.\int_{0}^{\infty} \mathcal{L}_{I_{u}}\left(\xi p^{-1} R^{\alpha(1-\epsilon)}\right)\right|_{R=r} f_{R}(r) d r \\
& =\int_{0}^{\infty} 2 \widetilde{\lambda} r e^{-\widetilde{\lambda} r^{2}} \mathcal{L}_{I_{u}}\left(\xi p^{-1} r^{\alpha(1-\epsilon)}\right) d r
\end{aligned}
$$


where $\widetilde{\lambda}=\pi \lambda$. We integrate from a point just outside the origin, to skip the typical BS that resides there, to $\infty$ where the closest UE can possibly exist.

Next, we will embark on finding $\mathcal{L}_{I_{u}}$, the Laplace transform of the distribution of the RV $I_{u}$. Substituting for $I_{u}$ from (11), gives

$$
\begin{aligned}
\mathcal{L}_{I_{u}}(s) & =\mathbb{E}\left[e^{-s I_{u}}\right] \\
& =\mathbb{E}\left[\exp \left(s \sum_{\mathfrak{z} \in \Psi}-p G_{\mathfrak{z}} R_{\mathfrak{z}}^{\alpha \epsilon} U_{\mathfrak{z}}^{-\alpha}\right)\right] \\
& =\mathbb{E}\left[\prod_{\mathfrak{z} \in \Psi} \exp \left(-s p G_{\mathfrak{z}} R_{\mathfrak{z}}^{\alpha \epsilon} U_{\mathfrak{z}}^{-\alpha}\right)\right]
\end{aligned}
$$

In (14), for each point $\mathfrak{z} \in \Psi$ there are three RVs: $G_{\mathfrak{z}}, R_{\mathfrak{z}}, U_{\mathfrak{z}}$. The $G_{\mathfrak{z}}$ are independent of the $R_{\mathfrak{z}}$ and of the $U_{\mathfrak{z}}$. However, $U_{\hat{z}}$ and $R_{\mathfrak{z}}$ are dependant in that $R_{\mathfrak{z}}<U_{\mathfrak{z}}$ (Recall that $R_{z}$ is the distance between an interfering UE $z$ and its typical BS, and $U_{\mathfrak{z}}$ is the distance between the same interfering UE $z$ and the typical BS at the origin). That is $\mathbb{P}\left[R_{\mathfrak{z}}<x \mid U_{\mathfrak{z}}=x\right]=1$, since if $U_{\mathfrak{z}}<R_{\mathfrak{z}}$ the interfering UE $\mathfrak{z}$ would associate with the typical $\mathrm{BS}$ at the origin.

Now we will resolve (14), as follows.

$$
\begin{aligned}
\mathcal{L}_{I_{u}}(s)= & \mathbb{E}_{\Psi, R_{3}, G_{z}}\left[\prod_{\mathfrak{z} \in \Psi} e^{-s p G_{\mathfrak{z}} R_{\mathfrak{z}}^{\alpha \epsilon} U_{\mathfrak{z}}^{-\alpha}}\right] \\
\stackrel{(\mathrm{a})}{=} & \mathbb{E}_{\Psi, R_{\mathfrak{z}}}\left[\prod_{\mathfrak{z} \in \Psi} \mathbb{E}_{G_{\mathfrak{z}}}\left[e^{\left.-s p G_{\mathfrak{z}} R_{\mathfrak{z}}^{\alpha \epsilon} U_{\mathfrak{z}}^{-\alpha}\right]}\right]\right. \\
\stackrel{(\mathrm{b})}{=} & \mathbb{E}_{\Psi, R_{\mathfrak{z}}}\left[\prod_{\mathfrak{z} \in \Psi} \int_{0}^{\infty} e^{-\left(1+s p R_{\mathfrak{z}}^{\alpha \epsilon} U_{\mathfrak{z}}^{-\alpha}\right) x} d x\right] \\
= & \mathbb{E}_{\Psi, R_{3}}\left[\prod_{\mathfrak{z} \in \Psi} \frac{1}{1+s p R_{\mathfrak{z}}^{\alpha \epsilon} U_{\mathfrak{z}}^{-\alpha}}\right]
\end{aligned}
$$

In (a) we used the fact that the $G_{\mathfrak{z}}$ are iid and in (b) we used the fact that $f_{G_{3}}(x)=e^{-x}$.

Next, we consider the expectation with respect to $\Psi$, to uncondition on $U_{\mathfrak{z}}$, the distance between every point $z \in \Psi$ and the origin. We will use for this expectation a PGFL, since $U_{\mathfrak{z}}$ is distributed differently for each point $\mathfrak{z} \in \Psi$.

Referring to Figure 1b, and based on our approximation, each point $z \in \Psi$ will be relocated to the position of the associated point $z \in \Phi$. That is, we will consider each interfering UE at point $z \in \Phi$ emitting power $p R_{3}^{\alpha \epsilon}$, but causing interference with this same power at the typical BS, at a distance $D_{z}$ based on the UE relocation. This allows us to write

$$
\mathbb{E}_{\Psi}\left[\prod_{\mathfrak{z} \in \Psi} f(\mathfrak{z})\right] \approx \mathbb{E}_{\Phi}\left[\prod_{z \in \Phi} f(z)\right]=e^{-\lambda \int_{\mathbb{R}^{2}}(1-f(x))} .
$$

Substituting for $f(y)$ from (15), converting to polar coordinates, and substituting for the angle integral by $2 \pi$, then

$$
\mathcal{L}_{I_{u}}(s)=\mathbb{E}_{R_{\mathfrak{\jmath}}}\left[\mathbb{E}_{\Phi}\left[\prod_{z \in \Phi} \frac{1}{1+s p R_{\mathfrak{z}}^{\alpha \epsilon} D_{z}^{-\alpha}}\right]\right]
$$

$$
=\mathbb{E}_{R_{3}}\left[e^{-2 \pi \lambda \int_{0}^{\infty} \frac{1}{1+(s p)^{-1} R_{3}^{-\alpha \epsilon_{x}}} x d x}\right]
$$

The distance $R_{\mathfrak{z}}$ is lower bounded by $U_{\mathfrak{\jmath}}$, for if $R_{\mathfrak{z}}<U_{\hat{\jmath}}$, the UE at $z$ would associate with the typical BS. But note that $U_{\mathfrak{z}}$ has been replaced now, through PP relocation, by $D_{3}$.

We will now apply the last expectation, $\mathbb{E}_{R_{3}}$, noting that the distribution of $R_{\mathfrak{z}}$ is Rayleigh. In light of (16), using the Rayleigh distribution $f_{R_{3}}(y)=2 \lambda \pi y e^{-\lambda \pi y^{2}}$, we have

$$
\begin{aligned}
\mathcal{L}_{I_{u}}(s) & =e^{-2 \pi \lambda \int_{0}^{\infty}\left(\mathbb{E}_{R_{3}}\left[\frac{1}{1+(s p)^{-1} R_{3}^{-\alpha \epsilon_{x} \alpha}}\right]\right) x d x} \\
& =e^{-2 \pi^{2} \lambda^{2} \int_{0}^{\infty} x \int_{0}^{x^{2}} \frac{e^{-\lambda \pi u}}{1+(s p)^{-1} u^{-\alpha \epsilon / 2} x^{\alpha}} d u d x}
\end{aligned}
$$

where $u=y^{2}$. Recall that $D_{z}^{-\alpha}$ and $R_{\mathfrak{z}}$ are dependent in that if $D_{z}=x$ then $R_{\mathfrak{z}}<x$. From (11) and (13), it follows that

$$
\begin{aligned}
\mathcal{L}_{I_{u}}\left(\xi p^{-1} r^{\alpha(1-\epsilon)}\right) & =e^{-2 \pi^{2} \lambda^{2} \int_{0}^{\infty} x \int_{0}^{x^{2}} \frac{e^{-\lambda \pi u}}{1+(s p)^{-1} u^{-\alpha \epsilon / 2} x^{\alpha}} d u d x} \\
& =e^{-2 \pi^{2} \lambda^{2} \int_{0}^{\infty} x \int_{0}^{x^{2}} \frac{\xi r^{\alpha(1-\epsilon)} e^{-\lambda \pi u}}{\xi r^{\alpha(1-\epsilon)}+u^{-\alpha \epsilon / 2} x^{\alpha}} d u d x} \text { (18) }
\end{aligned}
$$

From (13) and [18), we get

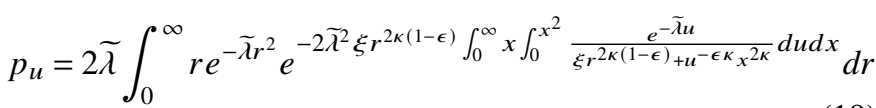

where $\widetilde{\lambda}=\pi \lambda$ and $\kappa=\alpha / 2$.

From (19), it appears that the UL coverage probability $p_{u}$ is dependent on the BS density $\lambda$. However, we will show in Theorem 2 below that the presence of $\lambda$ in 19 is superfluous.

Theorem 2: Under the stochastic geometric model of the cellular UL system, the UL coverage probability $p_{u}$ is independent of the BS density $\lambda$.

Proof: The proof is attained through a sequence of changes of variables. Starting with 19 , use the substitution $v=r^{2}$ to get

$$
p_{u}=\tilde{\lambda} \int_{0}^{\infty} e^{-\tilde{\lambda} v} e^{-2 \tilde{\lambda}^{2} \xi v^{\kappa(1-\epsilon)}} \int_{0}^{\infty} x \int_{0}^{x^{2}} \frac{e^{-\widetilde{\lambda} u}}{\xi v^{\kappa(1-\epsilon)}+u^{-\epsilon \kappa} x^{2 \kappa}} d u d x d v
$$

Use $y=x^{2}$ to get

$$
p_{u}=\tilde{\lambda} \int_{0}^{\infty} e^{-\widetilde{\lambda} v} e^{-\widetilde{\lambda}^{2} \xi v^{\kappa(1-\epsilon)} \int_{0}^{\infty} \int_{0}^{y} \frac{e^{-\tilde{\lambda} u}}{\xi v^{\kappa(1-\epsilon)}+u^{-\epsilon \kappa} y^{\kappa}} d u d y} d v
$$

Use $x=\tilde{\lambda} u$ to get

$$
p_{u}=\tilde{\lambda} \int_{0}^{\infty} e^{-\tilde{\lambda} v} e^{-\tilde{\lambda} \xi v^{\kappa(1-\epsilon)} \int_{0}^{\infty} \int_{0}^{\tilde{\lambda} y} \frac{e^{-x}}{\xi v^{\kappa(1-\epsilon)}+\left(\frac{x}{\bar{\lambda}}\right)^{-\epsilon \kappa}} d x d y} d v
$$

Use $z=\widetilde{\lambda} v$ to get

$$
p_{u}=\int_{0}^{\infty} e^{-z} e^{-\tilde{\lambda} \xi z^{\kappa(1-\epsilon)} \int_{0}^{\infty} \int_{0}^{\tilde{\lambda} y} \frac{e^{-x}}{\xi z^{\kappa(1-\epsilon)}+x^{-\epsilon \kappa}(\tilde{\lambda} y)^{\kappa}} d x d y} d z
$$

Finally, use $u=\tilde{\lambda} y$ to get

$$
p_{u}=\int_{0}^{\infty} e^{-z\left(1+\xi z^{\kappa(1-\epsilon)-1} \int_{0}^{\infty} \int_{0}^{u} \frac{e^{-x}}{\xi z^{\kappa(1-\epsilon)+x^{-\epsilon \kappa} u^{\kappa}}} d x d u\right)} d z
$$




\section{CONCLUSIONS}

In this article we have proven two theorems that go against established belief in the SG cellular modelling literature. Namely, we have proved that under the stochastic geometric model, the coverage probability in either DL and UL is independent of the BS density. This finding calls for a revisit to a large body of published results to rid them of a superfluous parameter.

\section{REFERENCES}

[1] M. Haenggi, Stochastic Geometry for Wireless Networks. Cambridge: Cambridge University Press, 2012.

[2] H. ElSawy, A. Sultan, M. Alouini, and M. Win, "Modeling and analysis of cellular networks using stochastic geometry: A tutorial," IEEE Communications Surveys and Tutorials, vol. 19, no. 1, pp. 167-203, 2017.

[3] S. Okegbile, B. Maharaj, and A. Alfa, "Stochastic geometry approach towards interference management and control in cognitive radio network: A survey," Computer Communications, vol. 166, no. 15, pp. 174-195, 2021.

[4] C. Liu, Y. Shen, and C. Lee, "Energy-efficient activation and uplink transmission for cellular iot," IEEE Internet of Things Journal, vol. 7, no. 2, pp. 906-921, 2020.

[5] X. Tang, X. Xu, and M. Haenggi, "Meta distribution of the sir in moving networks," IEEE Transactions on Communication, vol. 68 , no. 6 , pp. 3614-3626, 2020.

[6] Q. Liu and Z. Zhang, "The analysis of coverage probability,ase and ee in heterogeneous ultra-dense networks with power control," Digital Communications and Networks, vol. 6, no. 4, pp. 524-533, 2020.

[7] M. Haroon, F. Muhammad, Z. Abbas, G. Abbas, N. Ahmed, and S. Kim, "Proactive uplink interference management for nonuniform heterogeneous cellular networks," IEEE Access, vol. 8, pp. 55501555123,2021

[8] N. Kouzayha, H. Elsawy, H. Dahrouj, and T. Al-Naffouri, "Meta distribution of downlink sir for binomial point processes," IEEE Wireless Communications Letters, vol. 10, no. 7, pp. 1557-1561, 2021.

[9] B. Blaszczyszyn, M. Haenggi, P. Keeler, and S. Mukherjee, Stochastic Geometry Analysis of Cellular Networks. Cambridge: Cambridge University Press, 2018.

[10] C. Lee, C. Shih, and Y. Chen, "Stochastic geometry based models for modeling cellular networks in urban areas, wireless networks," Wireless networks, vol. 19, no. 6, pp. 1063-1072, 2013.

[11] J. Andrews, F. Baccelli, and R. Ganti, "A tractable approach to coverage and rate in cellular networks," IEEE Transactions on Communicationsy, vol. 59, no. 11, pp. 3122-3134, 2011.

[12] T. Bai and R. Heath, "Coverage and rate analysis for millimeter-wave cellular networks," IEEE Transactions on Wireless Communications, vol. 14, no. 2, pp. 1100-1114, 2015.

[13] J. Chen and C. Yuan, "Coverage probability and average rate of downlink user-centric wireless cellular networks with composite $\kappa-\mu$ shadowed and lognormal shadowed fading," IET Communication, vol. 13, no. 17, pp. 2805-2813, 2019.

[14] . Liu, J. Baudais, and P. Mary, "A tractable coverage analysis in dynamic downlink cellular networks," in 2020 IEEE 21 st International Workshop on Signal Processing Advances in Wireless Communications (SPAWC), pp. 1-5, IEEE, 2020

[15] M. Ouamri, M. Oteşteanu, A. Isar, and M. Azni, "Coverage, handoff and cost optimization for $5 \mathrm{~g}$ heterogeneous network," Physical Communication, vol. 39 , no. 5,2020 .

[16] J. Lei, H. Chen, and F. Zhao, "Stochastic geometry analysis of downlink spectral and energy efficiency in ultradense heterogeneous cellular networks," Mobile Information Systems, vol. 2018, 2018.

[17] M. Fadouol, "Rate and coverage analysis in multi-tier heterogeneous network using stochastic geometry approach," Ad Hoc Network, vol. 98, 2020.

[18] Y. Gao, S. Yang, S. Wu, M. Wang, and X. Song, "Coverage probability analysis for mmwave communication network with absf-based interference management by stochastic geometry," IEEE Access, vol. 7, pp. 133572-133582, 2019.

[19] S. Ali, M. Aslam, and I. Ahmed, "Uplink coverage probability and spectral efficiency for downlink uplink decoupled dense heterogeneous cellular network using multi-slope path loss model," Telecommunication systems, vol. 72, no. 4, pp. 505-516 1, 2019.
[20] J. Andrews, A. Gupta, and H. Dhillon, "A primer on cellular network analysis using stochastic geometry," ArXiv, 2016.

[21] A. Kundu, R. Pal, M. Kumar, and T. Sreejith, "Uplink and downlink performance bounds for full duplex cellular networks," in 2020 IEEE International Black Sea Conference on Communications and Networking (BlackSeaCom), (Odessa, Ukrain), 2020.

[22] E. Sadeghabadi, S. Abarghouyi, B. Makki, M. Kenari, and T. Svensson, "Asynchronous downlink massive mimo networks: A stochastic geometry approach," IEEE Transactions on Wireless Communications, vol. 19, no. 1, pp. 579-594, 2020.

[23] X. Wang and M. Gursoy, "Coverage analysis for energy-harvesting uavassisted mmwave cellular networks," IEEE Journal on Selected Areas in Communications, vol. 37, no. 12, pp. 2832-2850, 2019.

[24] P. Herath, C. Tellambura, and W. Krzymień, "Coverage probability analysis of three uplink power control schemes: Stochastic geometry approach," EURASIP journal on wireless communications and networking, vol. 1, pp. 1-14, 2018.

[25] N. Kouzayha, Z. Dawy, J. Andrews, and H. ElSawy, "Joint downlink/uplink rf wake-up solution for iot over cellular networks," IEEE Transactions on Wireless Communications, vol. 17, no. 3, pp. 1574 $1588,2018$.

[26] H. Mariam, I. Ahmed, and M. Aslam, "Coverage probability of uplink millimeter wave cellular network with non-homogeneous interferers' point process," Physical Communication, vol. 45, 2021.

[27] X. Jia, Q. Fan, W. Xu, and L. Yang, "Cross-tier dual-connectivity designs of three-tier hetnets with decoupled uplink/downlink and global coverage performance evaluation,” IEEE Access, vol. 7, pp. 16816-168361, 2019.

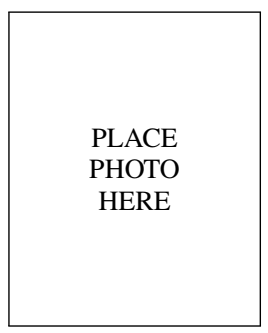

H. Nassar Prof. H. Nassar is professor in the Computer Science Department, Suez Canal University, Egypt. He received his B.Sc. in Electrical Engineering, from Ain Shams University, Egypt, in 1979. He received his MSc and $\mathrm{PhD}$ in Electrical Engineering from New Jersey Institute of Technology, USA, in 1985 and 1989, respectively. His research interests include wireless communications, stochastic geometry, queueing theory and artificial intelligence.

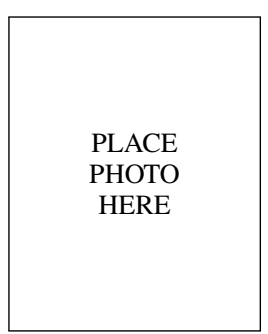

G. Taher Ms G. Taher is teaching assistant in the Computer Science Department, Suez Canal University, Egypt. She received her BSc and MSc in Computer Science from the same University in 2003 and 2015 respectively. Her research interests include IoT, Wireless Networks, Modeling and Simulation.

PLACE

PHOTO

HERE

S. El-Hady Dr. S El-hady is assistant professor in the Basic Sciences Department, Suez Canal University, Egypt. He received his BSc and MSc in Mathematics from the same University in 2004 and 2010 respectively. He received his $\mathrm{PhD}$ in Mathematics from Innsbruck University, Austria in 2016. His research interests include mathematical modeling, functional equations, and stability of functional, differential, and fractional differential equations. 